\title{
Sub Microsecond Notching of a Negative Hydrogen Beam at Low Energy Utilizing a Magnetron Ion Source with a Split Extractor
}

\author{
Douglas P. Moehs \\ Fermi National Accelerator Laboratory ${ }^{a}$, P.O. 500, Batavia IL 60510 USA
}

\begin{abstract}
A technique for sub-microsecond beam notching is being developed at $20 \mathrm{keV}$ utilizing a Magnetron ion source with a slit extraction system and a split extractor. Each half of the extractor is treated as part of a $50 \mathrm{ohm}$ transmission line which can be pulsed at \pm 700 volts creating a 1400 volt gradient. This system along with the associated electronics is electrically floated on top of a pulsed extraction voltage. A beam reduction of $95 \%$ has been observed at the end of the Fermilab $400 \mathrm{MeV}$ Linac and 35\% notching has recently been achieved in the Booster.
\end{abstract}

\section{INTRODUCTION}

Expanding neutrino research at Fermilab has created a demand for $8 \mathrm{GeV}$ proton intensities greater than the existing Proton Source (Linac and Booster) has ever provided [1]. Increases in proton intensity are presently limited by component activation associated with proton losses at high energies. Several projects have been initiated to address this problem including realignment, new magnets, and beam collimation in the Booster. As part of this effort, beam notching at low energies is being developed specifically to reduce the losses associated with beam injection and extraction from one accelerator to the next.

Effort to cleanly notch low energy beams has persisted, despite complications associated with space charge [2], due to the low beam rigidity and the relatively low cost associated with fast kilovolt pulsed-power supplies. At the SNS, a pulsed electric quadrupole in the $65 \mathrm{keV}$ LEBT is being used to create chopped beams [3]. At KEK a modulated surface-plasma converter source has modulated beams with 70 ns rise and fall times [4]. Furthermore, a traveling wave chopper utilizing special timing for a 35 $\mathrm{keV}$ beam was presented at the LINAC 2002 conference [5]. The split extractor technique presented here is complementary to these efforts. It is hoped that low energy space charge problems will be avoided by notching in the extraction region where the beam is expected to be space charge limited, due to the strong electric field of the extractor. The high gas pressure inherent to this region should also facilitate fast space charge recovery.

\footnotetext{
${ }^{a}$ Work Supported by University Research Associates Inc., contract number DE-AC02-76CH03000
} 


\section{EXPERIMENTAL SETUP}

A Magnetron ion source with a slit extraction system is used at Fermilab for the production of $\mathrm{H}$ minus ions [6]. Ions are extracted from the source at $20 \mathrm{keV}$, magnetically bent through 90 degrees and then further accelerated through a $750 \mathrm{kV}$ column. In order to deflect the ion beam without adding additional electrodes to the cramped source region the extractor has been split down the middle. Figure 1 shows the Fermilab magnetron ion source and the split extractor. The $30 \mathrm{kV}$ standoff and electrical feed through, on the left, replaced an observation window. Glass beads are now used to insulate the cables inside the vacuum instead of the Kapton tubing, shown in figure 1, which would turn greenish-black after several month of use and start to conduct along its exposed surfaces.

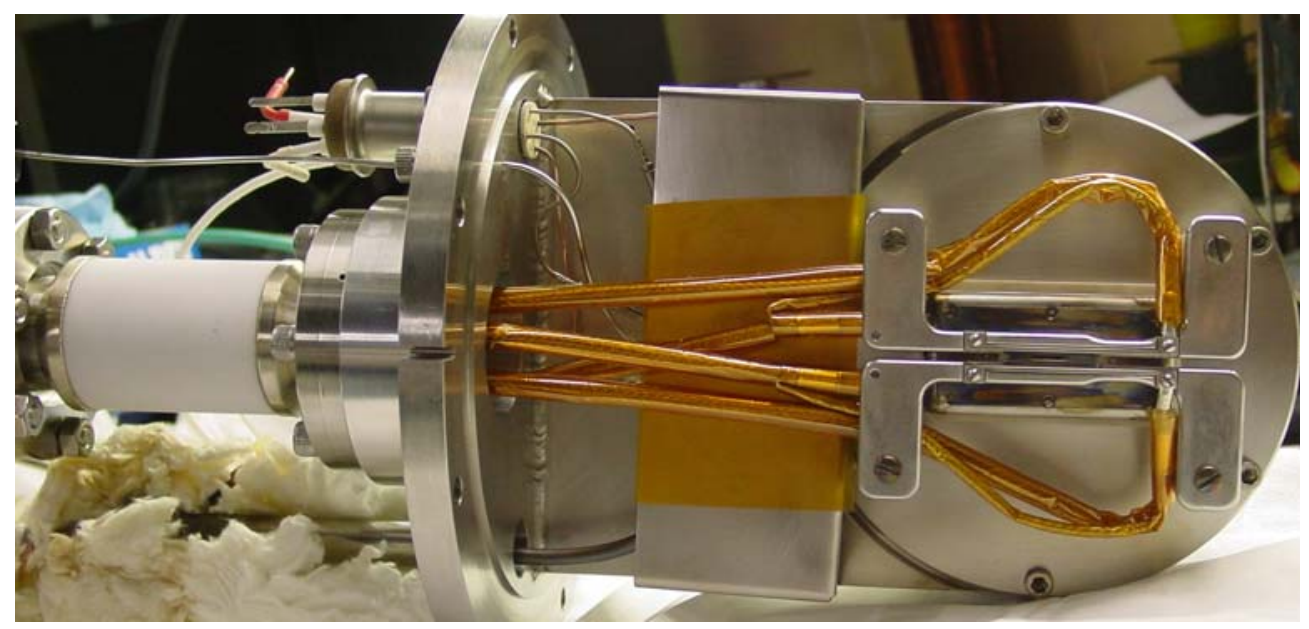

FIGURE 1. A picture of the split extractor used to extract $\mathrm{H}$ minus ions from the magnetron. The extractor components are mounted on $2.54 \mathrm{~cm}$ ceramic standoffs and are aligned by eye with the anode slit. The Kapton tubing shown here has been replaced by glass beads in recent experiments.

The tip of the extractor and the anode slit are aligned by eye and separated by approximately $2 \mathrm{~mm}$ using ceramic standoffs as mounting posts. The extractor slit width is also set to $2 \mathrm{~mm}$ using a feeler gauge. The anode slit is currently $0.7 \times 10$ $\mathrm{mm}$.

Beam notching is accomplished by treating each half of the extractor as part of a 50 ohm transmission line which can be pulsed at \pm 700 volts creating a 1400 volt gradient across the split extractor. Electrically the extraction electrodes appear as a $10 \mathrm{~cm}$ break in the transmission line. A 20 ns electrical pulse in standard 50 ohm coax has about a $4 \mathrm{~m}$ wave front so the extractor component of the transmission line should be invisible to the pulse. To verify this assumption, the reflected power was measured using TTL pulses and time domain reflectromitry. A reflected power of 5-10\% was measured. This result was strongly coupled to the connection quality at the extraction electrodes. The polarity of the voltage gradient is significant and is selected in order to optimize beam notch.

The \pm 700 volt high-voltage (HV) pulser, the 50 ohm loads and the vacuum feed through are electrically floated on top of the pulsed extraction voltage, typically 20 
$\mathrm{kV}$. The gate for the HV pulser is provided by a fiber optic network producing TTL pulses of the desired width. At least 3 Booster buckets, each 26 ns in width, are required to fire the Booster extraction magnet so the notch should be at least $78 \mathrm{~ns}$ wide.

The effective beam transition time can be computed by summing the electrical transition time of the $\mathrm{HV}$ pulser, the ion transit time through the extractor, and the curved path through the 90 degree bending magnet. The beam dynamics for a pair of deflection plates is described with more detail in reference [7]. An in house, dualpolarity high-voltage FET power supply was built for this application. Occasional sparking of the extraction system does not appear to be a problem for this supply. The electrical rise times are around 25 ns while the 55 ns fall times are slower than expected. Due to path length differences in the magnet, ions extracted from the source simultaneously will exit the magnet at different times. A SIMION model of the magnet suggests that $20 \mathrm{keV}$ ions are dispersed in time by roughly $10 \mathrm{~ns}$. Adding the above times to the $5 \mathrm{~ns}$ ion transit time through the extractor, $9.5 \mathrm{~mm}$ wide along the beam path, gives effective beam fall and rise times of $40 \mathrm{~ns}$ and $70 \mathrm{~ns}$ respectively.

\section{ACCELERATOR RESULTS AND DISCUSSION}

Figure 2 shows a beam trace from a Pearson current transformer with a 50 ns response time in the $750 \mathrm{keV}$ beam transport line. In this case, 8 notches were created to match the number of turns being injected into the Booster. The burst frequency is set to match the Booster

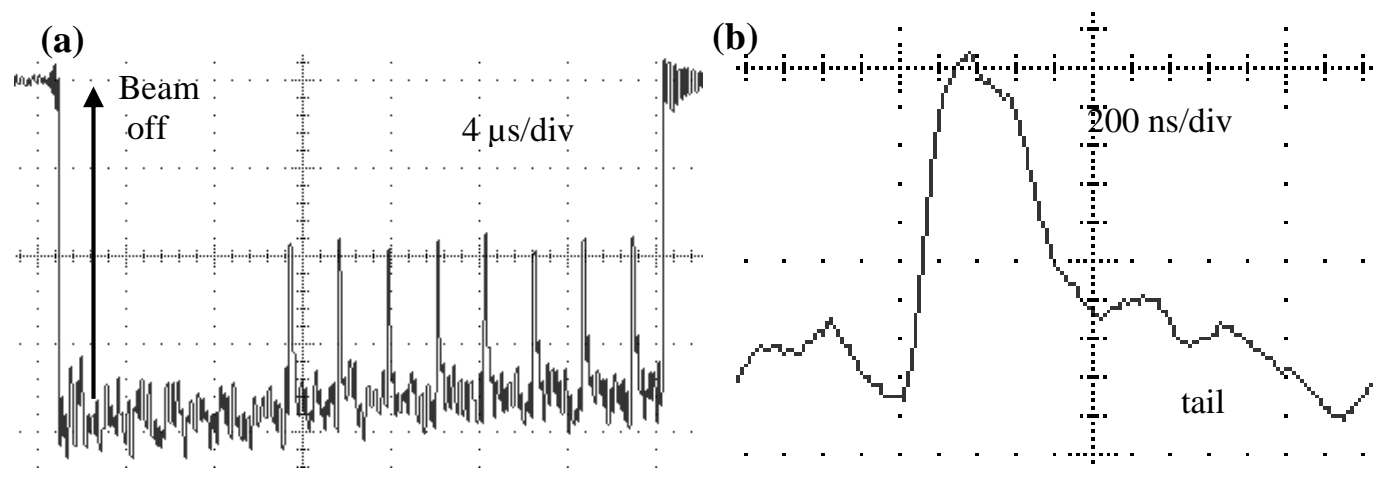

FIGURE 2. (a) A $20 \mathrm{~mA}$ beam of $\mathrm{H}$ minus with $50 \%$ notching observed at $750 \mathrm{keV}$ using a Pearson current transformer. (b) The rise and fall times of one notch is magnified in figure b.

injection frequency of $454 \mathrm{kHz}$. The applied voltage gradient at this time was 1200 volts, limited by resistors in the HV supply. In figure 2, the notches are 100 ns wide with approximately 40 ns and 70 ns rise and fall times respectively, as expected. The droop in beam intensity over the notching period, in this case about $1.5 \mathrm{~mA}$, is associated with an extended tail in the beam recovery time of each notch. The cause of this tail is not yet understood. Theories being investigated include electrode charging, meniscus disruption and recovery, and beam space charge recovery. 
The low energy end of the Fermilab Linac [8] operates at $200 \mathrm{MHz}$. Once the $\mathrm{H}$ minus ions are bunched and captured in the Linac the beam structure should be retained. A Beam Position Monitor (BPM) was used to measure one notch at $10 \mathrm{MeV}$. The raw BPM signal as observed by a fast oscilloscope is shown in figure 3 . The beam current is proportional to $1 / 2$ the signal strength so the notch depth at $10 \mathrm{MeV}$ is $50 \%$ as expected.

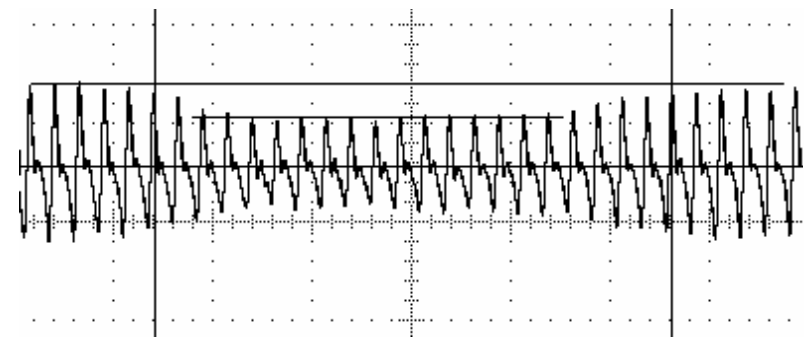

FIGURE 3. The raw BPM signal of a single notch measured at $10 \mathrm{MeV}$. Vertical and horizontal lines are added to guide the eye. The RF frequency is $200 \mathrm{Mhz}$ and the horizontal scale is $20 \mathrm{~ns} / \mathrm{div}$.

Figure 4(a) shows the first 4 turns of charge being injected into the Booster as measured by a toroid. The notch appears to grow with each injection. Figure 4(b) shows the signal from a phase detector in the Booster and compares notched and unnotched beam at $400 \mathrm{MeV}, 2.4 \mu$ into the machine cycle, just before the Booster extraction magnet would fire creating the traditional Booster extraction gap. In turn 4, right side of figure $4 \mathrm{a}$, the notch appears to be about 50\%, however in figure $4 \mathrm{~b}$ the beam reduction is only 35\%. During charge injection into the Booster the Booster RF is turned down allowing the $200 \mathrm{MHz}$ linac structure to fade prior to rebunching at 38 $\mathrm{MHz}$ for acceleration to $8 \mathrm{GeV}$. Thus the notch is adversely affected by the longitudinal space charge forces and in this case a reduction of $15 \%$ in notching efficiency was observed.

(a)

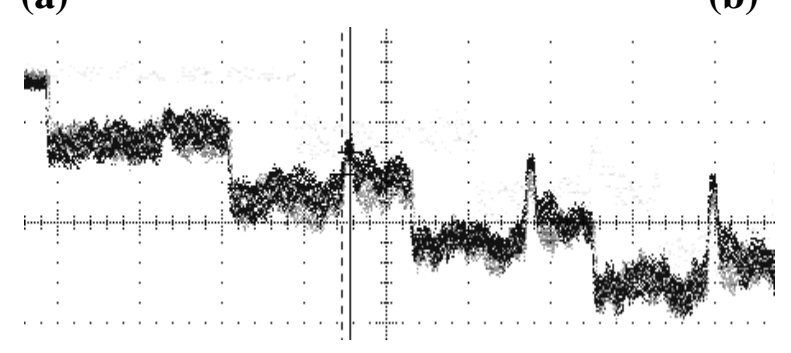

(b)

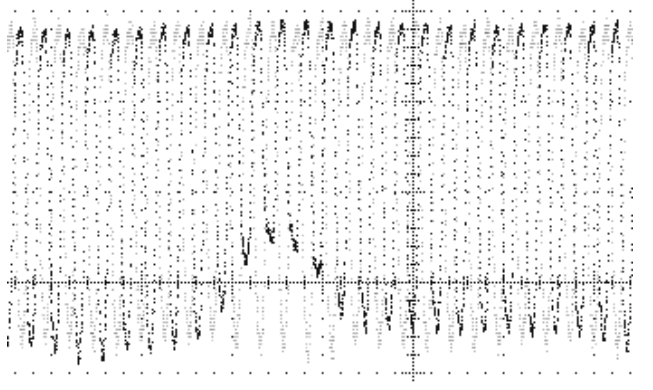

FIGURE 4. (a) Toroid signal of the first 4 turns of charge being injected into the Booster with the notch present on the left of each step. (b) Phase detector signal showing the Booster notch at $400 \mathrm{MeV}$, before acceleration. The lighter trace shows an un-notch Booster cycle.

In this experiment, only a trigger was available from the Booster. Thus the $100 \mathrm{~ns}$ pulse train, shown in figure 2 , was created by a pulse generator in burst mode. The frequency of the pulse generator was adjusted to optimize the notch efficiency. Better timing and a cleaner notch at $750 \mathrm{keV}$ should help improve the notching efficiency in the Booster. Once implemented, the notch will be timed in reducing the amount of beam kicked out at $4 \mathrm{MeV}$. 
An attempt has also been made to reduce the losses at the Linac switching magnet, where beam is sent to the Booster. A 150 ns notch was created and timed in using BPM's near the switching magnet at $400 \mathrm{MeV}$. Under normal operating conditions, a reduced activation rate of $25 \%$ was observed at the front end of the switching magnet. In this experiment, a beam reduction of $95 \%$ was measured at $400 \mathrm{MeV}$, during the notch, while the reduction at $750 \mathrm{keV}$ was only 50\%. This suggests that under certain conditions ions may continue to fall out of the notches as they propagate down the Linac. Efforts to scrape the beam in the $750 \mathrm{keV}$ line have begun in hopes of improving the low energy notch.

\section{ACKNOWLEDGEMENTS}

The author would like to thank James Wendt for his expert modifications of the ion source, Raymond Hren and Brian Stanzil for their ready help with cabling and electronics, Chris Jesnsen, for developing the FET HV pulser, James Lackey for help with TTL time domain reflectromitry, Milorad Popovic for accelerator assistance, Raymond Tomlin for his assistance with the BPM systems, William Pellico and Richard Meadowcroft for providing and fixing the fiber optic network and triggers, and Charles Schmidt for stimulating discussion and positive encouragement!

\section{REFERENCES}

1. Fermilab Proton Committee Report, www.fnal.gov/directorate/program_planning/studies/ProtonReport.pdf (2003).

2. J.G. Alessi, Rev. Sci. Instrum, 61, 1 (1990).

3. S. Nath, J. Billen, J. Stovall, H. Takeda, L. Young, Proceedings of LINAC 2002, p. 130, Korea (2002).

4. K. Shinto, A. Takagi, K, Ikegami, and Y. Mori, Rev. Sci. Instrum. 71, 696 (2000).

5. A. Novikov-Borodin, LINAC Conference Proceedings, Korea, 2002, pp. 115.

6. D. Moehs, "Studies on a Magnetron" in Production and neutralization of Negative Ions and Beams, edited by M. Stockli, AIP Conference Proceedings 639, Gif-sur-Yvette, France, 2002, pp. 115.

7. M. Stockli, US Particle Accelerator School Lecture notes: "Ion Beam Chopping”, Williamsburg VA (2004).

8. M. Popovic, L. Allen and C. W. Schmidt., Particle Accelerator Conference Proceedings, 1995, pp. 917. 